\title{
OPEN Endoscopic injection sclerotherapy improves liver function compared with endoscopic variceal ligation
}

\author{
Tsuguru Hayashi $^{\bowtie}$, Tatsuyuki Watanabe, Michihiko Shibata, Shinsuke Kumei, Shinji Oe, \\ Koichiro Miyagawa, Yuichi Honma \& Masaru Harada
}

Liver function is a most important prognostic factor in patients with liver cirrhosis. Also, portal hypertension is a fatal complication of liver cirrhosis and variceal treatment is indispensable. However, changes of liver functions after endoscopic variceal treatments are unknown. The aim of this study was to evaluate prognosis and liver functions after endoscopic injection sclerotherapy (EIS) and endoscopic variceal ligation (EVL). A total of liver cirrhotic 103 patients who underwent prophylactic EIS and EVL were enrolled. Overall survival rate was higher in EIS group than EVL group $(p=0.03)$. Multivariate analysis showed that EIS was a negative factor for death (HR: $0.46,95 \%$ confidence interval: $0.24-0.88, p=0.02$ ). Liver functions were assessed by blood test taken at before and 3 months after treatment. In EIS group, albumin and prothrombin time improved $(p<0.01)$, leading to improvement of Child-Pugh score, ALBI score and MELD score $(p<0.05)$. However, these did not improve in EVL group. EIS was a significant factor related to the elevated value of albumin after treatment in linear regression analysis (estimated regression coefficient: $0.17,95 \%$ confidence interval: $0.05-0.29$, $\mathrm{p}=0.005$ ). These results revealed that EIS could improve liver functions and prognosis.

Hemorrhage from gastroesophageal varices is one of the most common and serious complications in patients with liver cirrhosis ${ }^{1}$. Although the mortality from variceal hemorrhage has markedly decreased in the last two decades, its overall in hospital mortality is still as high as $16.8 \%^{2}$. Therefore, regular endoscopic observation is desirable ${ }^{3,4}$. If the gastroesophageal varices become large, prophylactic endoscopic therapies such as endoscopic injection sclerotherapy (EIS) or endoscopic variceal ligation (EVL) are recommended before the varices rupture ${ }^{5}$.

Several studies have compared EIS with EVL in terms of recurrence, rebleeding and prognosis ${ }^{6-10}$. EIS is superior to EVL in variceal recurrence rate ${ }^{7,8}$. However, EVL is easier to perform and its rate of complication is less than that of EIS ${ }^{9}$. Both endoscopic methods have different strong points. Thus, it is difficult to determine which prophylactic treatment should be selected.

Liver functions is an important factor that influences prognosis in patients with advanced chronic liver disease $^{11}$. In patients with hepatocellular carcinoma (HCC), treatment options are limited depending on the liver functions ${ }^{12-14}$. However, no study have compared EIS with EVL from the viewpoint of liver functions. Clarification of liver functions after EIS and EVL is therefore an issue of major importance.

We researched prognosis and long-term changes of liver functions after prophylactic endoscopic therapies for gastroesophageal varices. The aim of this study was to compare EIS with EVL in regard to prognosis and changes of liver functions after treatments.

\section{Results}

Patient characteristics. A total of 127 patients underwent prophylactic EIS and/or EVL. Among them, patients with no follow up $(n=17)$ and incomplete data $(n=7)$ were excluded from the study. Therefore, a total of 103 patients were enrolled in this study. The number of patients in the EIS and EVL groups was 64 and 39, respectively. The variceal forms were F2 or F3, and red wale sign was positive in all patients. Their baseline characteristics were shown in Table1. No factors differed between the two groups. All patients were performed as primary prophylaxis for variceal bleeding. No patients started nucleotide analogs for hepatitis B virus (HBV) and direct-acting antiviral agents for hepatitis $\mathrm{C}$ virus $(\mathrm{HCV})$ within 3 months after endoscopic treatments. 


\begin{tabular}{|l|l|l|l|}
\hline & EIS $(\mathbf{n}=\mathbf{6 4})$ & EVL (n= 39) & p value \\
\hline Age, years & $66(28$ to 85$)$ & $67(43$ to 87$)$ & 0.68 \\
\hline Male $(\%)$ & 68.8 & 64.1 & 0.67 \\
\hline BMI, $\mathrm{kg} / \mathrm{m}^{2}$ & $23.2(18.1$ to 38.9$)$ & $23.1(16.7$ to 37.9$)$ & 0.77 \\
\hline Etiology $(\mathrm{HBV} / \mathrm{HCV} / \mathrm{NBNC})$ & $12 / 15 / 37$ & $2 / 12 / 25$ & 0.14 \\
\hline Albumin, $\mathrm{g} / \mathrm{dL}$ & $3.5(2.5$ to 4.2$)$ & $3.5(2.3$ to 4.2$)$ & 0.32 \\
\hline Bilirubin, $\mathrm{mg} / \mathrm{dL}$ & $1.1(0.3$ to 2.9$)$ & $1.2(0.3$ to 3.1$)$ & 0.052 \\
\hline AST, $\mathrm{U} / \mathrm{L}$ & $39(17$ to 90$)$ & $42(13$ to 91$)$ & 0.80 \\
\hline ALT, $\mathrm{U} / \mathrm{L}$ & $28(10$ to 86$)$ & $28(6$ to 86$)$ & 0.89 \\
\hline Cre, $\mathrm{mg} / \mathrm{dL}$ & $0.76(0.43$ to 1.42$)$ & $0.80(0.41$ to 4.26$)$ & 0.55 \\
\hline eGFR, $\mathrm{mL} / \mathrm{min} / 1.73 \mathrm{~m}^{2}$ & $70.7(31.1$ to 161$)$ & $69.0(13.0$ to 119$)$ & 0.52 \\
\hline PT, $\%$ & $69.9(38.7$ to 97.9$)$ & $68.6(47.5$ to 100.0$)$ & 0.97 \\
\hline WBC, $/ \mu \mathrm{L}$ & $3900(1200$ to 8100$)$ & $3700(1100$ to 6600$)$ & 0.08 \\
\hline HGB, $\mathrm{g} / \mathrm{dL}$ & $11.8(7.0$ to 13.0$)$ & $11.3(7.1$ to 14.3$)$ & 0.19 \\
\hline PLT, $\times 10^{4} / \mu \mathrm{L}$ & $8.9(2.6$ to 20.3$)$ & $8.4(2.5$ to 26.1$)$ & 0.41 \\
\hline Child-Pugh score & $7(5$ to 9$)$ & $7(5$ to 9$)$ & 0.36 \\
\hline Fib-4 Index & $5.5(2.0$ to 21.8$)$ & $6.4(1.5$ to 19.2$)$ & 0.27 \\
\hline ALBI score & $-2.18(-2.82$ to -1.28$)$ & -2.09 (-2.79 to -0.89$)$ & 0.13 \\
\hline MELD score & $6(-1$ to 13$)$ & $6(2$ to 21$)$ & 0.22 \\
\hline Past history of HCC $(\%)$ & 31.2 & 41.0 & 0.40 \\
\hline Use of non-selective beta blockade $(\%)$ & 7.8 & 5.1 & 0.71 \\
\hline
\end{tabular}

Table 1. Baseline characteristics in EIS and EVL group. $\mathrm{p}$ values are results that compared EIS group with EVL group. Categorical variables were analyzed using $\chi^{2}$-test or Fisher's exact test, and continuous variables were compared using Mann-Whitney's $U$ test. alfa fetoprotein, $A L B I$ albumin-bilirubin, $A S T$ aspartate transaminase, $A L T$ alanine aminotransferase, $B M I$ body mass index, Cre creatinine, $e G F R$ estimated glomerular filtration rate, EIS endoscopic injection sclerotherapy, $E V L$ endoscopic variceal ligation, $H B V$ hepatitis B virus, $H C C$ hepatocellular carcinoma, $H C V$ hepatitis $\mathrm{C}$ virus, $H G B$ hemoglobin, $M E L D$ the model for end-stage liver disease, NBNC non-hepatitis B virus and non-hepatitis C virus, PLT platelet, $P T$ prothrombin time, $W B C$ white blood cell.

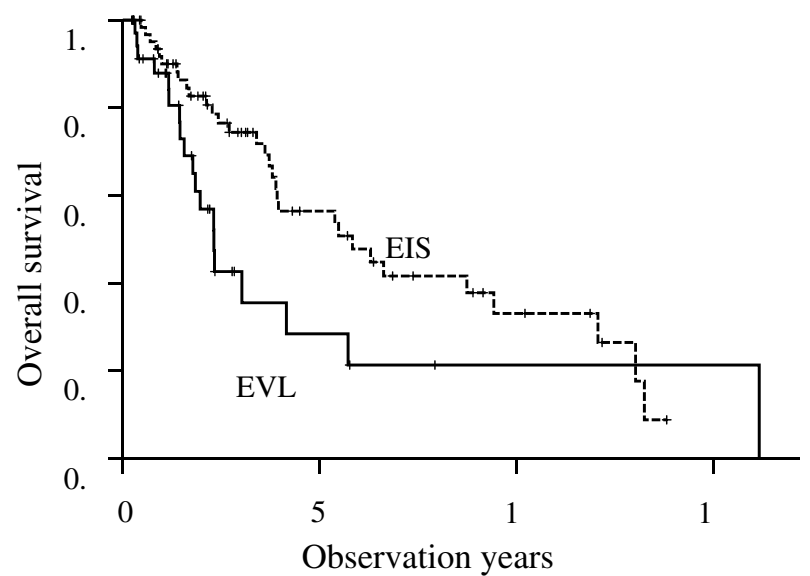

Figure 1. Overall survival in patients in the EIS group (dotted line) and EVL group (solid line). The EIS group showed a better prognosis than the EVL group ( 5 years survival rate: $56.5 \%$ vs $28.4 \%, \mathrm{p}=0.03$ ).

Prognosis after prophylactic variceal treatment. The median follow-up period of the 103 patients was $2.2(0.3-16.2)$ years and 5 years overall survival rate was $47.9 \%$. Stratified to method of treatments, 5 years survival rate of the EIS group was significantly longer than that of the EVL group (56.5\% vs $28.4 \%, \mathrm{p}=0.03$ ) (Fig. 1). In univariate analysis, age, etiology, Child-Pugh grade B, EIS, and past history of HCC were significant influencing factors for death. In multivariate analysis, Child-Pugh grade B (HR: 2.72, 95\% confidence interval (CI): $1.39-5.32, p=0.004)$, EIS (HR: $0.46,95 \%$ CI: $0.24-0.88, p=0.02)$ and past history of HCC (HR: $2.33,95 \%$ CI: $1.20-4.51, \mathrm{p}=0.01$ ) were significant influencing factors for death (Table 2 ). 


\begin{tabular}{|c|c|c|c|c|c|c|}
\hline & \multicolumn{3}{|c|}{ Univariate analysis } & \multicolumn{3}{|c|}{ Multivariate analysis } \\
\hline & HR & $95 \% \mathrm{CI}$ & p-value & HR & 95\% CI & p-value \\
\hline \multicolumn{7}{|c|}{ Age, years } \\
\hline$\leq 65$ & 1 & & & 1 & & \\
\hline$>65$ & 1.85 & $1.04-3.32$ & 0.04 & 1.75 & $0.93-3.29$ & 0.08 \\
\hline \multicolumn{7}{|l|}{ Gender } \\
\hline Male & 1 & & & & & \\
\hline Female & 1.24 & $0.67-2.29$ & 0.50 & & & \\
\hline \multicolumn{7}{|l|}{ BMI } \\
\hline$\leq 23.8$ & 1 & & & & & \\
\hline$>23.8$ & 1.01 & $0.56-1.79$ & 0.99 & & & \\
\hline \multicolumn{7}{|c|}{ Etiology } \\
\hline HBV & 1 & & & 1 & & \\
\hline $\mathrm{HCV}$ & 3.08 & $1.02-9.34$ & 0.047 & 2.28 & $0.72-7.19$ & 0.16 \\
\hline NBNC & 1.82 & $0.63-5.22$ & 0.27 & 1.57 & $0.49-5.07$ & 0.45 \\
\hline \multicolumn{7}{|c|}{ Child-Pugh grade } \\
\hline A & 1 & & & 1 & & \\
\hline $\mathrm{B}$ & 1.66 & $1.30-2.13$ & $<0.001$ & 2.72 & $1.39-5.32$ & 0.004 \\
\hline \multicolumn{7}{|c|}{ Treatment } \\
\hline EVL & 1 & & & 1 & & \\
\hline EIS & 0.52 & $0.29-0.95$ & 0.03 & 0.46 & $0.24-0.88$ & 0.02 \\
\hline \multicolumn{7}{|c|}{ Past history of HCC } \\
\hline No & 1 & & & 1 & & \\
\hline Yes & 2.86 & $1.59-5.14$ & $<0.001$ & 2.33 & $1.20-4.51$ & 0.01 \\
\hline
\end{tabular}

Table 2. Cox regression analysis about baseline factors associated with all-cause death in all patients. $B M I$ body mass index, CI confidence interval, EIS endoscopic injection sclerotherapy, HBV hepatitis B virus, HCC hepatocellular carcinoma, $H C V$ hepatitis $\mathrm{C}$ virus, $N B N C$ non-hepatitis $\mathrm{B}$ virus and non-hepatitis $\mathrm{C}$ virus.

\begin{tabular}{|c|c|c|c|c|c|c|}
\hline & \multicolumn{3}{|l|}{ EIS group } & \multicolumn{3}{|l|}{ EVL group } \\
\hline & Baseline & After treatment & $p$ value & Baseline & After treatment & $p$ value \\
\hline Albumin, g/dL & 3.5 (2.5 to 4.2$)$ & $3.6(2.7$ to 4.5$)$ & 0.002 & 3.5 (2.3 to 4.2$)$ & 3.4 (1.9 to 4.2$)$ & 0.17 \\
\hline Bilirubin, mg/dL & $1.1(0.3$ to 2.9$)$ & $1.1(0.3$ to 4.7$)$ & 0.87 & $1.2(0.3$ to 3.1$)$ & $1.2(0.5$ to 3.0$)$ & 0.82 \\
\hline AST, IU/L & 39 (17 to 90$)$ & $39(20$ to 104$)$ & 0.81 & 42 (13 to 91$)$ & 42 (17 to 93$)$ & 0.19 \\
\hline ALT, IU/L & $28(10$ to 86$)$ & 27 (6 to 92$)$ & 0.49 & $28(6$ to 86$)$ & $26(7$ to 132$)$ & 0.48 \\
\hline Cre, $\mathrm{mg} / \mathrm{dL}$ & $0.76(0.43$ to 1.42$)$ & 0.71 (0.46 to 1.48$)$ & 0.12 & $0.77(0.41$ to 4.26$)$ & $0.76(0.41$ to 1.00$)$ & 0.24 \\
\hline $\begin{array}{l}\mathrm{eGFR}, \mathrm{mL} / \\
\mathrm{min} / 1.73 \mathrm{~m}^{2}\end{array}$ & $70.7(31.1$ to 161$)$ & 73.0 (36.7 to 157$)$ & 0.09 & $69.7(13.0$ to 119$)$ & $64.7(11.0$ to 116$)$ & 0.33 \\
\hline PT, \% & 69.9 (38.7 to 97.9$)$ & $70.5(12$ to 100$)$ & $<0.001$ & $68.6(47.5$ to 100.0$)$ & $65.2(31$ to 100$)$ & 0.38 \\
\hline Child-Pugh score & $7(5$ to 9$)$ & $6(5$ to 11$)$ & 0.02 & $7(5$ to 9$)$ & $7(5$ to 11$)$ & 0.040 \\
\hline ALBI score & $\begin{array}{l}-2.18(-2.82 \text { to } \\
-1.28)\end{array}$ & $\begin{array}{l}-2.34(-2.99 \text { to } \\
-1.04)\end{array}$ & 0.003 & $\begin{array}{l}-2.09(-2.79 \text { to } \\
-0.89)\end{array}$ & $\begin{array}{l}-1.99(-2.73 \text { to } \\
-0.53)\end{array}$ & 0.069 \\
\hline MELD score & $6(-1$ to 13$)$ & $6(-1$ to 22$)$ & 0.04 & $6(2$ to 21$)$ & $6(2$ to 23$)$ & 0.43 \\
\hline
\end{tabular}

Table 3. Liver functions at baseline and 3 months after variceal treatments. $A L B I$ albumin-bilirubin, $A L T$ alanine aminotransferase, AST aspartate transaminase, Cre creatinine, eGFR estimated glomerular filtration rate, EIS endoscopic injection sclerotherapy, EVL endoscopic variceal ligation, $M E L D$ the model for end-stage liver disease, $P T$ prothrombin time.

Conversely, 3 years rebleeding or retreatment rate was not different between the two groups (EIS: $32.5 \%$ vs EVL: $26.7 \%, \mathrm{p}=0.18)$.

Liver function after prophylactic endoscopic variceal treatment. Albumin and prothrombin time improved significantly after treatment in the EIS group $(\mathrm{p}<0.01)$, but not in the EVL group (Table 3$)$. In the EIS group, Child-Pugh score, ALBI score and MELD score significantly improved after 3 months. In contrast, albumin and prothrombin time did not improve in the EVL group. Other liver functions, renal functions and blood count were not changed significantly in EIS and EVL groups.

The rate of change in Child-Pugh grade after variceal treatment is shown in Fig. 2. Among patients with Child-Pugh grade A at baseline, 90.6\% $(n=29)$ in the EIS group and $70.6 \%(n=12)$ in the EVL group maintained 


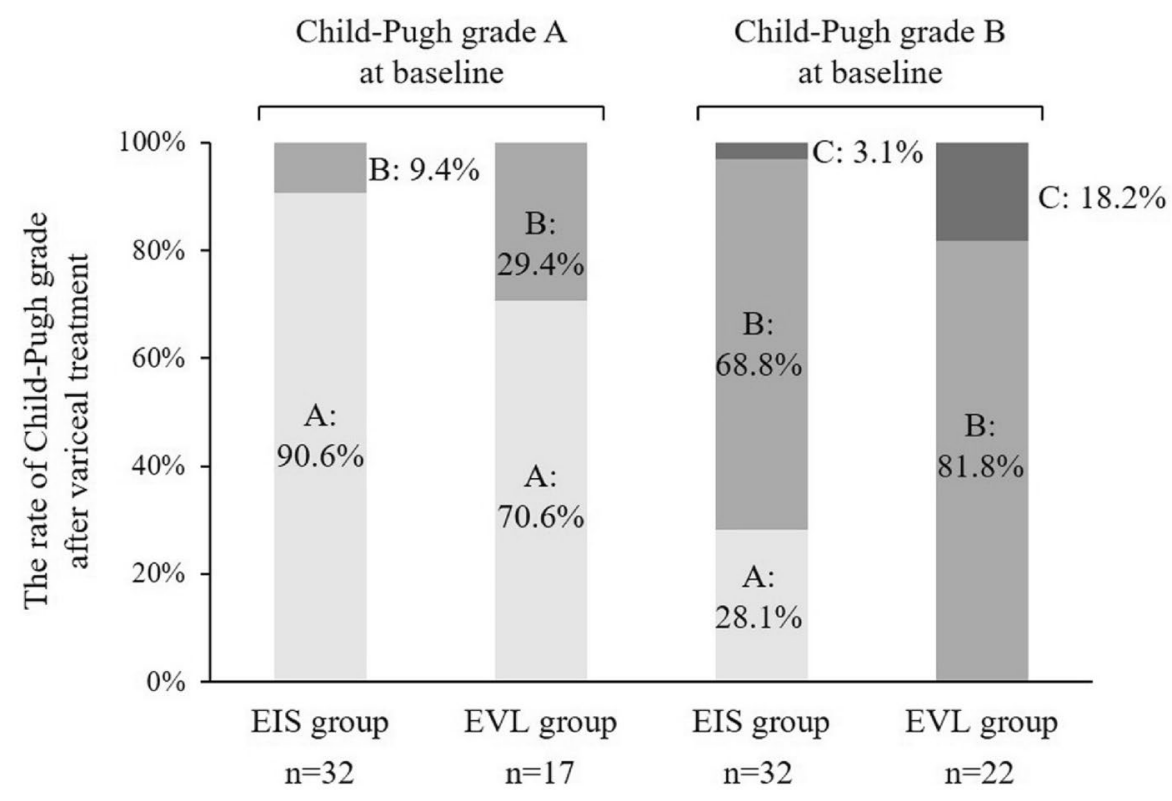

Figure 2. Change of Child-Pugh grade before and after variceal treatments. In patients with Child-Pugh grade $\mathrm{A}$, the rate of maintaining Child-Pugh grade A was higher in the EIS group ( 90.6 vs $70.6 \%, \mathrm{p}=0.11)$. In patients with Child-Pugh grade B, the rate of improving to Child-Pugh grade A was significantly higher in the EIS group (28.1 vs $0.0 \%, \mathrm{p}=0.015)$.

\begin{tabular}{|l|l|l|l|}
\hline & Estimated regression coefficient & $\mathbf{9 5 \%}$ CI & p-value \\
\hline Age, years (>65) & -0.02 & -0.14 to 0.10 & 0.79 \\
\hline Gender (male) & -0.06 & -0.19 to 0.06 & 0.33 \\
\hline BMI (>23.8) & 0.02 & -0.10 to 0.15 & 0.72 \\
\hline Etiology (NBNC) & 0.03 & -0.09 to 0.15 & 0.62 \\
\hline Child-Pugh (B) & 0.02 & -0.10 to 0.13 & 0.80 \\
\hline Treatment (EIS) & 0.17 & 0.05 to 0.29 & 0.005 \\
\hline Past history of HCC (present) & -0.08 & -0.21 to 0.04 & 0.18 \\
\hline
\end{tabular}

Table 4. Linear regression analysis about factors associated with increasing of serum albumin levels after variceal treatment in all patients. BMI body mass index, $C I$ confidence interval, EIS endoscopic injection sclerotherapy, $E V L$ endoscopic variceal ligation, $H B V$ hepatitis B virus, $H C C$ hepatocellular carcinoma, $H C V$ hepatitis $\mathrm{C}$ virus, $N B N C$ non-hepatitis $\mathrm{B}$ virus and non-hepatitis $\mathrm{C}$ virus.

Child-Pugh grade A, respectively. More importantly, $28.1 \%$ of patients of Child-Pugh grade B $(\mathrm{n}=32)$ improved to Child-Pugh grade A $(n=9)$. The rate of Child-Pugh improvement from B to A was significantly higher in the EIS group $(\mathrm{p}=0.015)$.

In linear regression analysis of elevated albumin levels after variceal treatments, EIS was a single independent predictor for improvement of albumin (estimated regression coefficient: $0.17,95 \%$ CI: $0.05-0.29, p=0.005$ ) (Table 4). Other factors were not significant.

\section{Discussion}

We first showed that the prognosis of EIS group was significantly better than EVL group. Second, we demonstrated that prophylactic EIS for gastroesophageal varices could significantly improve liver functions, whereas EVL did not improve liver functions. In particular, the rate of patients whose liver function increased from Child-Pugh grade B to A was higher in EIS group. In addition, linear regression analysis revealed that elevated albumin level after variceal treatment was associated with EIS.

A large number of studies have compared EIS with EVL ${ }^{6-10}$. EIS occludes the blood flow that supplies esophageal varices with sclerosing agent. This is the reason why EIS shows a lower recurrence rate of gastroesophageal varices than $\mathrm{EVL}^{7,9}$. Therefore, EIS is selected as a first choice of prophylactic variceal treatment in Japan. However, prognosis after variceal treatments and factors associated with prognosis are still unknown. Therefore, we studied the prognosis and liver functions after variceal treatments. This is the first study that compared EIS with EVL from the viewpoint of liver functions. Our study showed that the course of liver functions differed after these two prophylactic variceal treatments. This could lead to significant better prognosis after EIS than EVL. 
Hepatic blood flow gradually decreases with progression of chronic liver disease, leading to decline in hepatic functions ${ }^{15-17}$. In particular, portal blood flow gradually decreases in inverse proportion to Child-Pugh class and indocyanine green test ${ }^{18,19}$. That results in decreasing transport of glucose, amino acid and fatty acid to hepatocytes and an increase in oxidative stress and liver inflammation. In response to decreased portal flow, hepatic arterial flow increases complementarily ${ }^{20,21}$. However, in hyperdynamic state, superior mesenteric artery and splenic arterial flow are increased and result in an increase of blood flow in the portal system. These hemodynamics causes development of collateral vessels, especially emergence of gastroesophageal varices. However, EIS changes the hepatic hemodynamics by occluding collateral blood flow. Takahashi et al. reported that portal venous flow increased after EIS ${ }^{22}$. The increase of blood flow in the liver sinusoid increases shear stress, which causes the release of a variety of cytokines such as interleukin (IL)-6, hepatocyte growth factor and nitric oxide from the sinusoidal endothelium ${ }^{23-25}$. These could induce hepatocyte proliferation. In fact, after portal vein embolization (PVE), which is a preoperative preparation for extensive liver resection, the volume of the non-embolized lobe was increased and the Ki-67 labelling index was higher in the non-embolized lobe ${ }^{26}$. These evidences suggest that the number of hepatocytes increased and liver regeneration occurred by repairing portal hemodynamics. This may lead improvement of liver functions.

The same mechanism is shown in balloon occluded retrograde transvenous obliteration (BRTO) and percutaneous transhepatic obliteration (PTO). Several previous studies reported that BRTO could increase portal venous flow and improve liver functions by obstruction of the portosystemic shunt ${ }^{27,28}$. However, not all patients who underwent BRTO showed improved liver functions ${ }^{29}$. Also in our study, some patients did not experience improved liver functions. The reason for poor response to increased portal flow is uncertain. We have to clarify this problems and other predictive factors for improvement of liver functions.

We demonstrated that EIS improved liver functions. However, we have to mention that patients with Child-Pugh grade $\mathrm{C}$ were not enrolled in this study. Patients with uncontrollable ascites or hyperbilirubinemia were not indicated for prophylactic EIS. When liver damage is advanced, EIS could cause liver failure and death in several days ${ }^{30}$. Therefore, it is important to consider appropriate indications for EIS.

This study has several limitations. First, we carried out this study with a small sample size. This could have an impact on statistics. Second, the present study was analyzed retrospectively. Third, selection of treatment method was not randomized. EVL may be performed in patients with poor general conditions. However, there was no difference between EIS and EVL groups at baseline in this study. Therefore, a prospective study with large number of patients should be performed to analyze prophylactic variceal treatments.

In conclusion, we demonstrated that EIS has the potential to improve liver functions, which could lead to a better prognosis than with EVL.

\section{Methods}

Patients. We retrospectively analyzed the liver cirrhotic patients who underwent prophylactic EIS and/or EVL from April 2002 to July 2020 in our hospital. The diagnosis of gastroesophageal varices was based on endoscopic findings at 1 month before variceal treatment. Endoscopic findings of esophageal varices were evaluated according to the grading system defined by the Japanese Research Committee on Portal Hypertension and outlined in the general rules for recording endoscopic findings of esophageal varices ${ }^{31}$. Varices were classified as F1: small and straight, F2: enlarged and tortuous, or F3: large and coil-sharped. EIS was performed on red wale sign positive or F2/F3 variceal patients. Patients with the following conditions were excluded from the study; (1) lost to follow up and (2) incomplete data. Patients with Child-Pugh grade C or major portal vein tumor thrombus were not included in this study, because they were not recommended for prophylactic variceal treatment. Regarding the selection of variceal treatments, EIS was our first choice and we changed perform EVL if it was difficult to do intravariceal injection. This study was conducted in accordance with the 1975 Declaration of Helsinki. This study was approved by the institutional review board of our hospital (the ethics committee name is ethics committee of university of occupational and environmental health, Japan and the code number is H29-079). This study is a retrospective observational study and gives no disadvantage to patients. Therefore, the ethics committee decided that informed consent is not required and waived. However, we announced publicly that patients could refuse to participate in this study if they desire.

\section{Endoscopic treatment}

EIS was performed using a flexible gastrointestinal endoscope (GIF Q260J: Olympus Optical, Tokyo, Japan) under fluoroscopy and a combination of intermittent intravariceal injection of 5\% ethanolamineoleate with iopamidol (5\% EOI). Oral side of the injection point was occluded by balloon, and we injected EOI retrogradely to supplying vessels. EIS was repeated weekly until disappearance of variceal form and red wale sign. When it was difficult to perform intravariceal injection, EVL was added.

EVL was performed using pneumo-activate EVL device (Sumius, Tokyo, Japan) and cylinders. Ligation bands were applied to varices in a step ladder pattern. This procedure was also repeated until disappearance of variceal form and red wale sign.

Argon plasma coagulation (APC) was added in both groups after EIS or EVL according to the judgement of endoscopic specialists.

Prognosis and liver function assessment. Clinical and laboratory information of patients was obtained from electronic medical records. Medical historical variables consisted of age, sex, body mass index (BMI), etiology of liver cirrhosis, Child-Pugh score and grade ${ }^{32}$, Fib-4 index ${ }^{33}$, ALBI score ${ }^{34}$, MELD score ${ }^{35}$, history of HCC and use of non-selective beta blockade. Overall survival (OS) and time to rebleeding or retreatment were compared between EIS and EVL groups. OS was duration time from the variceal treatment to death from any 
cause or last follow-up. Blood variables included liver functions test (serum total bilirubin, albumin, aspartate aminotransferase, alanine aminotransferase, gamma-glutamyl transpeptidase, prothrombin time), renal functions (serum creatinine, estimated glomerular filtration rate) and complete blood count at baseline and 3 months after endoscopic treatment.

Statistical analysis. Baseline parameters were compared between the EIS and EVL groups using MannWhitney $U$ test and $\chi^{2}$ test. Baseline data comparing the EIS group and EVL group were shown as median value (minimum to maximum values). The Kaplan-Meier method with log rank test was used to analyze the prognosis and the Cox proportional hazard model was used for univariate and multivariate analysis of factors for prognosis. $p$ values were calculated for all tests, with a value of $p<0.05$ considered to be statically significant. Changes of liver functions after EIS or EVL were compared using the Wilcoxon single-rank sum test and Fisher's exact test. Linear regression analyses were performed to identify the factors associated with elevated serum albumin levels. We defined change of serum albumin levels as differences from baseline to 3 months after treatment. All statistical analyses were performed using the Statistical Package for the Social Science (SPSS) version 25 (SPSS Inc., Chicago, IL, USA) and Easy R (EZR) version 1.29 (Saitama Medical center, Jichi Medical University, Saitama, Japan), and graphical use interface for R (The R Foundation for Statistical Computing, Vienna, Austria) ${ }^{36}$.

\section{Data availability}

All relevant data are within the manuscript.

Received: 25 February 2021; Accepted: 27 September 2021

Published online: 14 October 2021

\section{References}

1. Garcia-Tsao, G. et al. Prevention and management of gastroesophageal varices and variceal hemorrhage in cirrhosis. Hepatology 46, 922-938 (2007).

2. Sato, M. et al. Variceal hemorrhage: Analysis of 9987 cases from a Japanese nationwide database. Hepatol. Res. 45, 288-293 (2015).

3. T. Hayashi et al., Use of the serum Wisteria floribunda agglutinin-positive Mac2 binding protein as a marker of gastroesophageal varices and liver-related events in chronic hepatitis C patients. Diagnostics (Basel) 10 (2020).

4. Tamaki, N. et al. Validation of albumin, bilirubin, and platelet criteria for avoiding screening endoscopy in patients with advanced fibrosis. Hepatol. Res. 50, 996-999 (2020).

5. Garcia-Tsao, G., Abraldes, J. G., Berzigotti, A. \& Bosch, J. Portal hypertensive bleeding in cirrhosis: Risk stratification, diagnosis, and management: 2016 practice guidance by the American Association for the study of liver diseases. Hepatology 65, 310-335 (2017).

6. Kitano, S., Koyanagi, N., Iso, Y., Higashi, H. \& Sugimachi, K. Prevention of recurrence of esophageal varices after endoscopic injection sclerotherapy with ethanolamine oleate. Hepatology $7,810-815$ (1987).

7. Hou, M. C. et al. Comparison of endoscopic variceal injection sclerotherapy and ligation for the treatment of esophageal variceal hemorrhage: A prospective randomized trial. Hepatology 21, 1517-1522 (1995).

8. Hou, M. C., Lin, H. C., Lee, F. Y., Chang, F. Y. \& Lee, S. D. Recurrence of esophageal varices following endoscopic treatment and its impact on rebleeding: Comparison of sclerotherapy and ligation. J. Hepatol. 32, 202-208 (2000).

9. Baroncini, D. et al. A prospective randomized trial of sclerotherapy versus ligation in the elective treatment of bleeding esophageal varices. Endoscopy 29, 235-240 (1997).

10. Svoboda, P., Kantorova, I., Ochmann, J., Kozumplik, L. \& Marsova, J. A prospective randomized controlled trial of sclerotherapy vs ligation in the prophylactic treatment of high-risk esophageal varices. Surg. Endosc. 13, 580-584 (1999).

11. Tamaki, N. et al. Non-invasive prediction of hepatocellular carcinoma development using serum fibrosis marker in chronic hepatitis C patients. J. Gastroenterol. 49, 1495-1503 (2014).

12. Hiraoka, A., Kumada, T., Michitaka, K. \& Kudo, M. Newly proposed ALBI grade and ALBI-T score as tools for assessment of hepatic function and prognosis in hepatocellular carcinoma patients. Liver Cancer 8, 312-325 (2019).

13. Kudo, M., Chung, H. \& Osaki, Y. Prognostic staging system for hepatocellular carcinoma (CLIP score): Its value and limitations, and a proposal for a new staging system, the Japan Integrated Staging Score (JIS score). J. Gastroenterol. 38, 207-215 (2003).

14. Hayashi, T. et al., Antiplatelet therapy improves the prognosis of patients with hepatocellular carcinoma. Cancers (Basel) 12 (2020).

15. Kobayashi, M. et al. Assessment of hepatic steatosis and hepatic tissue blood flow by xenon computed tomography in nonalcoholic steatohepatitis. Hepatol. Res. 39, 31-39 (2009).

16. Takahashi, H. et al. Evaluation of quantitative portal venous, hepatic arterial, and total hepatic tissue blood flow using xenon CT in alcoholic liver cirrhosis: Comparison with liver cirrhosis C. Alcohol Clin. Exp. Res. 31, S43-48 (2007).

17. Ikeda, H. et al. Xenon computed tomography shows hemodynamic change during the progression of chronic hepatitis C. Hepatol. Res. 37, 104-112 (2007).

18. Annet, L. et al. Hepatic flow parameters measured with MR imaging and Doppler US: Correlations with degree of cirrhosis and portal hypertension. Radiology 229, 409-414 (2003).

19. Van Beers, B. E. et al. Hepatic perfusion parameters in chronic liver disease: Dynamic CT measurements correlated with disease severity. AJR Am. J. Roentgenol. 176, 667-673 (2001).

20. Gulberg, V., Haag, K., Rossle, M. \& Gerbes, A. L. Hepatic arterial buffer response in patients with advanced cirrhosis. Hepatology 35, 630-634 (2002).

21. Lautt, W. W. \& Greenway, C. V. Conceptual review of the hepatic vascular bed. Hepatology 7, 952-963 (1987).

22. Takahashi, H. et al. Xenon computed tomography can evaluate the improvement of hepatic hemodynamics before and after endoscopic injection sclerotherapy. J. Gastroenterol. 48, 1353-1361 (2013).

23. Schoen, J. M., Wang, H. H., Minuk, G. Y. \& Lautt, W. W. Shear stress-induced nitric oxide release triggers the liver regeneration cascade. Nitric Oxide 5, 453-464 (2001).

24. Marubashi, S. et al. Effect of portal hemodynamics on liver regeneration studied in a novel portohepatic shunt rat model. Surgery 136, 1028-1037 (2004).

25. Nagino, M. et al. Changes in hepatic lobe volume in biliary tract cancer patients after right portal vein embolization. Hepatology 21, 434-439 (1995).

26. Tranchart, H. et al. Liver regeneration following repeated reversible portal vein embolization in an experimental model. Br. J. Surg. 103, 1209-1219 (2016).

27. Miyamoto, Y., Oho, K., Kumamoto, M., Toyonaga, A. \& Sata, M. Balloon-occluded retrograde transvenous obliteration improves liver function in patients with cirrhosis and portal hypertension. J. Gastroenterol. Hepatol. 18, 934-942 (2003). 
28. Kumamoto, M. et al. Long-term results of balloon-occluded retrograde transvenous obliteration for gastric fundal varices: Hepatic deterioration links to portosystemic shunt syndrome. J. Gastroenterol. Hepatol. 25, 1129-1135 (2010).

29. Yamamoto, A. et al. Prediction for improvement of liver function after balloon-occluded retrograde transvenous obliteration for gastric varices to manage portosystemic shunt syndrome. J. Vasc. Interv. Radiol. 27, 1160-1167 (2016).

30. Nishioka, K. et al. Extreme hyperbilirubinemia induced by endoscopic injection sclerotherapy in a patient with esophageal varices and thalassemia: Report of a case. Surg. Today 26, 53-56 (1996).

31. Japanese Research society for Portal Hypertension. The general rules for recording endoscopic findings of esophageal varicesRevised edition. Acta Hepatol. Jpn. 33, 271-281 (1991).

32. Pugh, R. N., Murray-Lyon, I. M., Dawson, J. L., Pietroni, M. C. \& Williams, R. Transection of the oesophagus for bleeding oesophageal varices. Br. J. Surg. 60, 646-649 (1973).

33. Sterling, R. K. et al. Development of a simple noninvasive index to predict significant fibrosis in patients with $\mathrm{HIV} / \mathrm{HCV}$ coinfection. Hepatology 43, 1317-1325 (2006).

34. Johnson, P. J. et al. Assessment of liver function in patients with hepatocellular carcinoma: A new evidence-based approach-the ALBI grade. J. Clin. Oncol. 33, 550-558 (2015).

35. Kamath, P. S., Kim, W. R., Advanced Liver Disease Study Group. The model for end-stage liver disease (MELD). Hepatology 45, 797-805 (2007).

36. Kanda, Y. Investigation of the freely available easy-to-use software "EZR" for medical statistics. Bone Marrow Transplant. 48, 452-458 (2013).

\section{Author contributions}

Conceptualization: T.H. and T.W.; Methodology: T.H.; Formal analysis: T.H.; Investigation: T.H.; Writing: T.H., T.W., M.S., O.S., K.M., S.K., Y.M., M.H.; Project administration: T.H., T.W., M.S., M.H. All authors have red and agreed to the published version of the manuscript.

\section{Funding}

This study was funded by Ministry of Education, Culture, Sports, Science and Technology (No. 18K15832).

\section{Competing interests}

The authors declare no competing interests.

\section{Additional information}

Correspondence and requests for materials should be addressed to T.H.

Reprints and permissions information is available at www.nature.com/reprints.

Publisher's note Springer Nature remains neutral with regard to jurisdictional claims in published maps and institutional affiliations.

(c) (i) Open Access This article is licensed under a Creative Commons Attribution 4.0 International License, which permits use, sharing, adaptation, distribution and reproduction in any medium or format, as long as you give appropriate credit to the original author(s) and the source, provide a link to the Creative Commons licence, and indicate if changes were made. The images or other third party material in this article are included in the article's Creative Commons licence, unless indicated otherwise in a credit line to the material. If material is not included in the article's Creative Commons licence and your intended use is not permitted by statutory regulation or exceeds the permitted use, you will need to obtain permission directly from the copyright holder. To view a copy of this licence, visit http://creativecommons.org/licenses/by/4.0/.

(c) The Author(s) 2021 